\title{
Standing on Their Own Two Feet: How the New Public Charge Rules Could Impact Non-European LPR Applicants
}

\author{
Kendal Lowrey ${ }^{1}$ (D) Jennifer Van Hook ${ }^{1}$
}

Received: 19 May 2020 / Accepted: 17 March 2021 / Published online: 31 March 2021

(c) The Author(s), under exclusive licence to Springer Nature B.V. 2021

\begin{abstract}
In February 2020, the U.S. government began to implement a new Public Charge rule that greatly expands the definition of "public charge" when determining admissibility for legal permanent residency (LPR). The rule seeks to determine not only whether applicants used public benefits in the past, but also whether they are likely to use them in the future. However, predicting future use based on characteristics measured at the time of application, such as English language proficiency and income, is difficult. We evaluate the risk of being deemed inadmissible as well as the likelihood of using public assistance by regional group. Using a sample of recently arrived LPRs in the 2013-2017 American Community Survey, we find that Mexicans/Central Americans face disproportionate risk of being deemed a public charge despite their relatively low public assistance use. This increased risk would likely alter the composition of newly admitted LPRs with relatively fewer Mexican/ Central American LPRs.
\end{abstract}

Keywords Immigrants $\cdot$ Public charge $\cdot$ Public assistance $\cdot$ Admissibility $\cdot$ Region National origin

\section{Introduction}

The possibility that immigrants might benefit from public assistance programs has inflamed debates about immigration for decades. Immigration restrictionists worry that the United States-with its high standard of living and relatively generous social service programs - has been a "welfare magnet" to poor people in other countries (Borjas, 1990; Borjas \& Hilton, 1996) despite the fact that noncitizens are ineligible for most types of public assistance (Fortuny and Chaudry, 2012) and use welfare at lower rates than the U.S. born (Capps et al., 2009). These concerns however, partially motivated the welfare reforms of the 1996 Personal Responsibility and Work

Kendal Lowrey

k11289@psu.edu

1 The Pennsylvania State University, University Park, PA 16802, USA 
Opportunity Reconciliation Act (PRWORA), which restricted noncitizen's eligibility for many federal public assistance programs and led to sharp reductions in immigrant public program use (Fix \& Passel, 1999). While economic conditions of the late 1990s may have also spurred reduction in use of public assistance (Bell, 2001; Lofstrom \& Bean, 2002), immigrants incurred chilling effects, leaving them fearful of using such aid in the case that doing so may be counted against them at some time in the future (Capps, 2001). In fact, Capps et al. (Capps, Fix, et al., 2020) find that, as a likely result of fear surrounding the public charge rules, participation in federal benefit programs have declined twice as fast among noncitizens relative to citizens between years 2016 and 2019. Nevertheless, new public charge provisions advanced by the prior administration sought to determine admissibility by predicting future use of public assistance based on past use of benefits and other characteristics measured when applying for Lawful Permanent Residency (LPR) status.

The Public Charge rules build upon prior acts that restrict immigrant admission to the U.S. Since 1882, applicants could be denied admission if they were judged to be at risk of becoming a public charge. Under the Clinton Administration, informal guidance was issued that limited the scope of the Public Charge provision yet left federal agencies to determine admissibility (Faber, 2018). However, the Trump Administration formalized the Rule, arguing that doing so helps to better carry out provisions of the original statute. The new Department of Homeland Security (DHS) and the Department of State (DOS) Public Charge rules expanded the definition of public charge to include a much wider set of federal public assistance programs and, moreover, employed a series of negative and positive factors to determine whether a person is likely to become a public charge at any point in their lifetime. Public assistance includes cash, food, housing, and health assistance to low-income populations. Public charge rules are not new, however, the revision to the rule encompassed a wider variety of restrictions and a forward-looking approach that may unfavorably disqualify LPR applicants coming with less favorable characteristics on the basis of their presumed future welfare need. In addition, the Rule's implementation during the Covid-19 pandemic raised serious public health concerns that immigrants may forego vital healthcare needs out of fear of utilizing potentially necessary resources.

In this paper, we analyze the potential disparate impacts of the new Public Charge rules on applicants by region of birth. We specifically focus on two parallel changes in the definition of "public charge" in the determination of admissibility of applications for legal permanent residency status, one implemented by the Department for Homeland Security (DHS) and another by the Department of State (DOS). Although these specific rules were temporarily blocked by court rulings and future viability of the new rules remains unknown under the Biden Administration, this research remains relevant. Actions such as these remain a policy goal of immigration restrictionists and therefore continue to shape debates about how to best reform U.S. immigration and admissions policy. We argue that the changes to the rule like those advanced by the prior administration could alter the immigrant selection process and shift the national origin composition of LPRs. Even though exclusionary policies have become much less explicitly based on race or national origin than in the past, changes to the Public Charge rule could favor admission of some regional groups over others. 


\section{Background}

The public charge rules were issued in 2019 by both the Department of Homeland Security (DHS) and the Department of State (DOS). These rules revise previous public charge criteria for determining the admissibility of applicants for legal permanent residency. DHS issued the Inadmissibility on Public Charge Grounds Final Rule on August 14, 2019. It applies to U.S. residents who seek to adjust their status. The Interim Final Rule was issued by the DOS (84 F.R. 54,996) on October 11, 2019. It was modeled on the DHS Rule, but applies to those who apply for admission outside the United States at a foreign consulate. Even though there remain several lawsuits contesting the DHS and DOS Public Charge Rules, the Supreme Court cleared the way for the DHS rule to be implemented in January 2020 while the cases work their way through the courts. As a consequence, DHS began to implement its rule on February 24, 2020, and it is likely that DOS will follow suit, further impacting those applying through a foreign consulate. Finally, as a consequence of the Covid-19 pandemic, a preliminary injunction suspended the Public Charge Rules on July 29, 2020, however a full stay of the injunction was granted on September 11, 2020, thereby reactivating the implementation of the Public Charge Rules despite the nation's public health crisis.

Both Rules are amendments to the Immigration and Nationality Act of 1952 (the INA). Under the INA Section 212(a)(4), inadmissibility based on public charge grounds has been determined by the statute's "totality of the circumstances" test (TOC), which includes, at minimum, consideration of the following factors: age; health; family status; assets, resources, and financial status; and education and skills. Additionally, prior use of federal cash public assistance programs could be grounds for denial. However, the new DHS and DOS rules greatly expand the list of public benefit programs to include consideration of food assistance (SNAP), federal housing assistance and federal public health insurance such as Medicaid. ${ }^{1}$

The new rules are also explicitly forward-looking in that they seek to determine not only whether an applicant has used an expanded set of public benefits in the past, but also whether they are more likely than not to use public benefits in the future. The new rules thus establish a set of positive and negative factors to be used to make this determination. Positive factors, for example, include earnings or household income above $250 \%$ of the poverty level and private health insurance coverage, while negative factors include public benefit use within the past 36 months, economic inactivity, diagnosis of a work-limiting health condition, low English proficiency, and low skills or education. Some of the negative factors are weighted more heavily than others, including having a health condition, economic inactivity and prior public benefits use. A full list of positive and negative factors is included in Table 1 (discussed later in further detail).

\footnotetext{
1 (1) Medicaid, with certain exceptions; (2) Supplemental Nutrition Assistance Program (SNAP); (3) Sect. 8 housing; (4) Sect. 8 Housing Assistance under the Housing Choice Voucher Program; (5) Sect. 8 Project-Based Rental Assistance; and (6) Federal Public Housing.
} 
Table 1 List of negative and positive factors in the public charge rule

Label Description

A. Heavily-weighted negative factors

(1) Economic Inactivity

The noncitizen is "not a full-time student and is authorized to work, but is unable to demonstrate current employment, recent employment history, or a reasonable prospect of future employment;'[1]

(2) Public Benefit Use:

The noncitizen has "received or has been certified or approved to receive one or more public benefits, as defined in $\S 212.21$ (b) [including Medicaid, Supplemental Nutrition Assistance Program (SNAP), Sect. 8 housing, Sect. 8 Project-Based rental assistance, Federal public housing, SSI, and TANF or other state income-support means-tested programs] for more than 12 months in the aggregate within any 36 month period prior to the...application;'[2]

(3) Health Condition

(4) Previous Public Charge Finding

B. Other negative factors *

(1) Low income

(2) Low education/skills

(3) Low English proficiency

(4) Age-related criteria

(5) Large household size

(6) Affidavit of Support

C. Heavily-weighted positive factors

(1) Household Income

The noncitizen "has been diagnosed with a medical condition that is likely to require extensive medical treatment or institutionalization or that will interfere with the alien's ability to provide for himself or herself, attend school, or work; and...is uninsured and has neither the prospect of obtaining private health insurance, or the financial resources to pay for reasonably foreseeable medical costs;"[3] and

The noncitizen "was previously found inadmissible or deportable on public charge grounds."[4]

Pursuant to the statute, age, health, family status, assets, resources, and financial status, and education and skills must also be considered when determining whether an applicant is "more likely than not" to become a public charge in the future. Specific negative factors include:

$<125 \%$ of FPG; $<100 \%$ of FPG for active armed forces personnel and their spouse and children; this measure excludes public assistance income

The Rule is unclear about the definition of low skills

The Rule is unclear about the definition of low English proficiency

Age 62 or older and having an income that is less than $125 \%$ of the FPG, not counting public assistance income $100 \%$ FPG is used as cut-off for armed services personnel and their spouse and children

The Rule is unclear about the meaning of large household

It is unlikely that the applicant's sponsor would be able to provide financial support to applicant; income of sponsor $<125 \%$ FPG

The noncitizen has a "household income, assets, or resources, and support... of at least 250 percent of the Federal Poverty Guidelines [(FPG)];'[5]

(2) Employment Income

The noncitizen is authorized to work and is currently employed in a legal industry with an annual income...250 percent of the Federal Poverty Guidelines [(FPG)] for the [applicant's] household size;"'[6] and 
Table 1 (continued)

\begin{tabular}{ll}
\hline Label & Description \\
\hline (3) Private Insurance & The noncitizen "has private health insurance...private health \\
& must be appropriate for the expected period of admission, and \\
& does not include health insurance for which the [applicant] \\
& receives subsidies in the form of premium tax credits under the \\
[ACA].”[7]
\end{tabular}

[1] 8 C.F.R. \$212.22(c)(1)(i); [2] 8 C.F.R. \$212.22(c)(1)(ii).; [3] 8 C.F.R. \$212.22(c)(1)(iii); [4] 8 C.F.R. \$212.22(c)(1)(iv); [5] 8 C.F.R. \$212.22(c)(2)(i); [6] 8 C.F.R. §212.22(c)(2)(ii); [7] 8 C.F.R. $\$ 212.22(\mathrm{c})(2)(\mathrm{iii})$

*Table 33 in Inadmissibility on Public Charge Grounds, 83 Fed. Reg. 126 (October 10, 2018) (to be codified at 8 CFR Parts 103, 212, 213, 214, 245 and 248)

As others have argued (e.g., Capps, Bachmeier, et al., 2018; Capps, Greenberg, et al., 2018), the forward-looking negative and positive factors are likely to have much greater impact on LPR admissions than the criteria related to past public benefits use. The reason is that the vast majority of foreign-born applicants would not have been able to use the programs in question anyway. They were either not living in the United States at the time of their application or, if they were living in the U.S. under a temporary visa or as an undocumented immigrant, they would have been categorically ineligible for such benefits. Despite their relatively low use, concerns about public program use by immigrant households have persisted, and pundits continue to claim that immigrants use welfare at very high rates (e.g., Arthur, 2018; Richwine, 2018), even though most assistance going to immigrant households takes the form of food assistance (e.g., SNAP and school meal programs) and in the case of undocumented immigrants, is designated only for the US-born children living in their households, which falls within the bounds of current law. Since the passage of the 1996 welfare reform, access to federally funded benefits has been limited to qualified immigrants who have been in the U.S. for 5 years or more; undocumented immigrants were ineligible for assistance even prior to the reform and remain ineligible. Furthermore, public assistance use among U.S.-born minor children of immigrants has been found to parallel their immigrant parents' receipt of services; U.S.-born children have less access to some of the benefit programs for which their parents are ineligible (Fomby \& Cherlin, 2004).

Finally, it is important to note that the new Rules are ambiguous. They direct officials to deny admission if the negative factors outweigh the positive factors, and that heavily weighted factors should be counted more than non-heavily weighted factors, and that a single negative factor is insufficient for an inadmissibility determination. However, they are unclear about how many of the different positive and negative factors should be measured (such as low English proficiency), and about the precise number or combination of positive and negative factors that will lead to an applicant being deemed inadmissible. This uncertainty adds to the anxiety felt by applicants. For example, it likely contributes to chilling effects whereby immigrants and their families forego participating in public 
assistance programs or seeking health care out of fear of it counting against them in future applications for immigration benefits (Batalova et al., 2018; Page et al., 2020; Perreira et al., 2018).

\section{Evaluating the Disparate Impacts of the Public Charge Rules}

In this paper, we ask two questions related to how the new public charge rules change the likelihood of being deemed inadmissible, leaving aside questions about chilling effects for other research.

First, we ask whether the new public charge rules have disparate impacts on the risk of inadmissibility across national origin groups, thus potentially altering the national origin composition of newly admitted legal immigrants. We already have a good sense of the answer to this question, so our contribution here is mostly to update earlier work on this topic. Capps et al. at the Migration Policy Institute (Capps, Greenberg, et al., 2018) analyzed recently arrived LPRs in the American Community Survey (ACS), whom they used as a proxy for future LPR applicants. When they conducted their study, the final Rules had not yet been made public, so they evaluated the likely impacts of the draft of the proposed DHS public charge rule that was leaked in January and March of 2018 by Vox. They found that the leaked rule could put a large share of applicants at risk of receiving a public charge determination. For example, 69 percent had at least one negative factor and 43 percent had two or more negative factors. The cut-off for a public charge determination is unclear, but these figures suggest that at least two in five would face non-negligible risk of being deemed inadmissible. Additionally, the leaked rule would have the potential of dramatically changing the national origin composition of immigrants who are granted LPR status because the application of negative and positive factors would lead to Mexicans and Central Americans being deemed inadmissible more often than other groups.

We extend the Capps, Greenberg, et al. (2018), study by providing an update of results after the final rule was introduced. We account for as many of the negative and positive factors described in the final rules as possible given data constraints. Additionally, we summarize the relative risk of inadmissibility based on the final Public Charge rules with a three-tiered inadmissibility risk scale, showing the possibility of a shift in the national origin composition of LPRs as a result of the implementation of the new Public Charge rules. Lastly, we separate public benefit use by gender and show how the risk of being deemed inadmissible differs between men and women.

Our second question concerns how effective the new Public Charge rules are at identifying those who are most likely to use public assistance. This is an important question because if the positive and negative factors are not predictive of future welfare use, it would undermine the official justification for the Rules and, moreover, could unfairly penalize groups that are socioeconomically disadvantaged or have low English proficiency but are not particularly likely to use public assistance programs. This portion of the analysis is novel; we are unaware of any prior work evaluating this aspect of the new Public Charge rules. As noted, the Public Charge rules use a 
list of positive and negative factors to identify applicants who are more likely than not to use public benefits in their lifetime. Yet it would be surprising if these factors were predictive because the need for public assistance can quickly change due to unforeseen circumstances (e.g., recession, layoffs, health crises). Some states may choose to provide public assistance to a broader range of immigrants than are covered by federally funded public assistance programs; immigrants may instead forego federal assistance in favor of state-level aid. Finally, the public charge determination is based on characteristics of immigrants measured at the time of application, before they have had time to adapt to American society and the U.S. labor force. Earnings and English language proficiency tend to improve with time in the United States (Alba \& Nee, 2003; Chiswick \& Miller, 2002; Myers \& Pitkin, 2010), meaning that early assessments of immigrants' prospects could quickly change.

The negative and positive factors may be particularly poor at predicting public benefit use among Mexicans and Central Americans. Indeed, past research conducted on welfare recipiency shows that immigrants, especially labor migrants from Mexico and Central America, have tended to use cash assistance less often than similarly-poor U.S. born adults (Bean et al., 1997; Van Hook \& Bean, 2009a). Moreover, Mexican immigrant women who are on public assistance tend to have shorter welfare spells and are more likely to leave welfare for work than their U.S.-born counterparts (Van Hook \& Bean, 2009b), a pattern that the authors attribute to this group's work-related cultural repertoires. This suggests that the factors identified in the Public Charge rules, such as being unemployed, low income, and low education, are likely to be particularly weak predictors of public assistance receipt among those from Mexico and Central America.

\section{Methodology}

\section{Data and Sample}

We rely primarily on data obtained from the 2013-2017 years of the American Community Survey (ACS) (Ruggles et al., 2020). The ACS is a large representative survey that is continuously conducted by the U.S. Census Bureau across all communities in the United States. An important strength of the ACS is that it has a very large sample size, and therefore, supports analyses of recently adjusted LPRs for small-sized national origin groups. Capps, Greenberg, et al. (2018) and Capps, Bachmeier, et al. (2018) also relied on the ACS for this reason.

To answer our first question about the impact of the Public Charge rules on the risk of inadmissibility, we examine the share with positive and negative factors among Lawful Permanent Resident (LPR) adults (age 18+) who were admitted or had adjusted status within the last 5 years. This group serves as a proxy for applicants who would be impacted by the public charge rules in the future, although they may have more favorable profiles than they had at the time of their admission given that they have had up to 5 years to learn English, gain education or training, and become more integrated into the labor force. Therefore, our decision to use recentlyadmitted LPRs as a proxy probably leads to conservative estimates of the risk of 
inadmissibility by the Rule. To answer our second question about the ability of the negative and positive factors to accurately identify those who will use public benefits, we examine public benefits use among LPR adults and naturalized citizens who were admitted or had adjusted status in the last 5-9 years. This sample restriction is important because LPRs are ineligible for most federal benefits programs in their first 5 years in the country, but they can become eligible after 5 years of residency, especially if they naturalize or work for 40 quarters.

We also relied on the 2008 and 2014 Survey of Income and Program Participation (SIPP) for supplemental analyses. The SIPP is a moderately sized Census survey designed in part to measure public assistance trends for the U.S. population. Despite its smaller sample size, the SIPP can provide useful insights about immigrants' history of public benefit use, which we used to justify certain assumptions we made about immigrants' public benefit use prior to admission as an LPR. Additionally, we used the 2008 SIPP as an auxiliary data source for imputing LPR status among the foreign-born in the ACS as described below. We also utilize the 2008 and 2014 SIPP together as a benchmark for ACS public program usage rates. Given that program usage is underreported in the ACS (Meyer et al., 2009), it is possible that our ACSbased analysis provides conservative estimates of risk of being deemed inadmissible and public benefit use.

LPRs are identified in the ACS using a unique imputation methodology as developed and validated by Van Hook and Bachmeier (Van Hook et al., 2015) and used by the Migration Policy Institute for their estimates (Capps, Bachmeier, et al., 2018). This methodology assigns noncitizens in the ACS an immigration status (naturalized citizen, LPR, other) by linking the ACS data to the 2008 Survey of Income and Program Participation, which includes a question on immigrants' legal status, using multiple imputation methods. For a more detailed description of this methodology, see Batalova, Sarah and Capps (2014). This methodology provides similar estimates of the unauthorized population by country of birth, age, sex, and duration of U.S. residence as the residual method used by the DHS (Bachmeier et al., 2014) and the Center of Migration Studies (CMS) (Warren, 2014) (see Capps, Gelatt, et al., 2020 for a demographic profile of the unauthorized population based on the methods used here). However, the imputation methodology is imperfect in that it is difficult to differentiate some temporary legal immigrants from LPRs and due to the nature of random variation in the imputation procedure, it is possible that the imputation status across imputations may vary. We impute five times and average across imputations to account for this variation.

We also exclude foreign-born persons who are exempt from the Public Charge rules (refugees, asylees, parolees, those admitted under a Special Immigrant Visa, Cuban and Haitian entrants and asylum seekers, NACARA, American Indians born in Canada) based on algorithms developed by the Migration Policy Institute (Batalova et al., 2014). We were unable to remove other exempt categories (e.g., VAWA, Amerasians, Special Immigrant Juveniles) because the ACS lacks the information necessary to identify them. After excluding the aforementioned groups, the sample (ACS) includes a large number of LPRs who were admitted 0-4 years ago $(N=130,905)$ and LPRs and naturalized citizens who were admitted 5-9 years ago $(N=94,515)$. 


\section{Positive and Negative Factors}

We created measures that indicate whether potential applicants have the negative and positive factors according to the Public Charge rules. Table 1 summarizes the negative and positive factors included in the rules that guide our analyses. We utilize several ACS measures to capture these factors (as described below). It is important to note that ACS survey data is limited by how the questions have been posed or categorized. For example, the Rules take into account credit scores, wealth, whether the applicant received a fee waiver, their relationship to their sponsor, and their sponsor's financial information when evaluating applications, but none of this information is available in the ACS. Additionally, the Rules do not provide clear guidance on the definition of many factors such as low English proficiency, low skills, or having a large household size. We followed the guidelines in the Rules whenever possible, but sometimes we had to rely on our own interpretations to operationalize the measures that the rules left ambiguous.

\section{Heavily Weighted Negative Factors}

We measure two heavily weighted negative factors: (1) having a health condition with no means of paying for the necessary medical care (i.e., having at least one chronic condition or functional limitation and not having private health insurance or an income that is $250 \%$ of the federal poverty guideline (FPG) or greater, not counting public assistance income); and (2) economic inactivity (defined as not attending school and not employed or in the armed forces among adults age $16+{ }^{2}$ ).

Of special note, the Public Charge rules count prior use of federal public programs $^{3}$ as a heavily weighted negative factor, specifically, whether the applicant received or has been certified or approved to receive benefits for more than 12 months within the 36 month period prior to the application. Nevertheless, we did not count public benefits use as a negative factor because the ACS public assistance/benefits measures do not capture participation during the relevant time period. Instead, they pertain to the 12 months prior to the interview. This omission is unlikely to affect the results because very few LPR applicants would have been eligible to have received federal public assistance. Our supplementary analyses of the 2008 SIPP confirm that fewer than 1 percent of adjustees received cash assistance prior to adjustment and virtually none of the new arrivals (those who applied for admission at a foreign consulate) had done so.

Despite this, we do use the ACS public assistance measures to address our second question about the predictive validity of the positive and negative factors. For this analysis, we do not need information on past receipt of public assistance. Instead,

\footnotetext{
${ }^{2}$ Excluding persons age $18+$ who are the parent of a pre-school child or who live with a parent with one or more functional limitations (primary care givers under the Rule).

3 Medicaid, Supplemental Nutrition Assistance Program (SNAP), Section 8 housing, Section 8 ProjectBased rental assistance, Federal public housing, SSI, and TANF or other state income-support meanstested programs.
} 
we assess the strength of the relationship between the risk of inadmissibility (based on the positive and negative factors) and current receipt of a federal cash program (SSI or TANF) or public health insurance coverage (Medicaid). In some analyses, we exclude food assistance (SNAP) because the ACS does not indicate which household members are receiving SNAP. It is possible that only the U.S.-born family members in a mixed-status immigrant household (e.g., children) are recipients, and the Rules exempt public benefit use by the U.S.-born family members of immigrants. Nevertheless, because immigrants benefit from living in a SNAP-receiving household even if they are not the direct beneficiary, we conducted additional analyses in which we included SNAP in our measure of public benefits use.

\section{Other Negative Factors}

The Public Charge rules also consider several other negative factors. We were able to measure five of them: (1) low income, defined as having income less than $125 \% 4$ of the federal poverty guideline (FPG) excluding public assistance income; (2) having low skills, which we defined as having less than a high school degree; (3) low English proficiency, which we defined as speaking English "not well" or "not at all"; (4) age-related criteria (being 62 or older and having an income that is less than $125 \%$ of the FPG, not counting public assistance income); and (5) large household size, which we defined as six or more persons, which is more than twice the average U.S. household size in 2017 (2.54).

\section{Heavily Weighted Positive Factors}

We measured three heavily- weighted positive factors: (1) high household income (250\% of FPG or higher, excluding public assistance income), (2) currently working with individual earnings greater than $250 \%$ of FPG for a single adult, and (3) private health insurance coverage.

\section{Three-Tier Inadmissibility Risk Scale}

We developed a three-tiered risk scale (high, medium, low) to summarize the number and weight of positive and negative factors. This scale gives greater weight to heavily weighted negative and positive factors than the other negative factors. It also takes into consideration how positive factors may offset negative factors, and it accounts for a statement in the Rules that a single negative factor would be insufficient for a public charge designation.

The high-risk group is defined as having a combination of at least one heavily weighted negative factor or two more other negative factors, and having no positive factors at all. As shown in Table 2, 10.6\% of the potential applicants in the highrisk group have a health condition, $42.2 \%$ are economically inactive, $61.4 \%$ are low

\footnotetext{
$4<100 \%$ of FPG for active armed forces personnel and their spouse and children.

$5100 \%$ FPG is used as cut-off for armed services personnel and their spouse and children.
} 
Table 2 Risk profiles among recently-arrived LPRs

\begin{tabular}{|c|c|c|c|c|c|c|}
\hline \multirow[t]{2}{*}{ Heavily-weighted Negative Factors } & \multicolumn{2}{|c|}{ High Risk } & \multicolumn{2}{|c|}{ Medium Risk } & \multicolumn{2}{|c|}{ Low Risk } \\
\hline & $\%$ & SE & $\%$ & SE & $\%$ & SE \\
\hline Health Condition & 10.6 & $(0.3)$ & 0.0 & $(0.0)$ & 0.0 & $(0.0)$ \\
\hline Economic Inactivity & 42.2 & $(0.4)$ & 35.1 & $(0.4)$ & 0.0 & $(0.0)$ \\
\hline \multicolumn{7}{|l|}{ Other Negative Factors } \\
\hline Low Income & 61.4 & $(0.4)$ & 21.0 & $(0.3)$ & 0.0 & $(0.0)$ \\
\hline Low Skill & 58.8 & $(0.5)$ & 28.5 & $(0.4)$ & 0.0 & $(0.0)$ \\
\hline Low English Proficiency & 75.7 & $(0.4)$ & 49.9 & $(0.5)$ & 0.0 & $(0.0)$ \\
\hline Age $\geq 2 \&$ Low Income & 8.3 & $(0.2)$ & 1.6 & $(0.1)$ & 0.0 & $(0.0)$ \\
\hline Large Household Size & 27.9 & $(0.6)$ & 22.5 & $(0.3)$ & 0.0 & $(0.0)$ \\
\hline \multicolumn{7}{|l|}{ Heavily-weighted Positive Factors } \\
\hline High HH Income & 0.0 & $(0.0)$ & 52.3 & $(0.4)$ & 68.1 & $(0.4)$ \\
\hline Earning $>250 \% \mathrm{FPL}$ & 0.0 & $(0.0)$ & 3.6 & $(0.1)$ & 27.7 & $(0.3)$ \\
\hline Private Health Insurance & 0.0 & $(0.0)$ & 53.7 & $(0.4)$ & 72.9 & $(0.4)$ \\
\hline Sample Size & 29,538 & & 49,054 & & 35,314 & \\
\hline
\end{tabular}

Source: 2013-2017 American Community Survey

See text for definitions of negative and positive Factors. High risk= no heavily weighted positive factors, and at least one heavily weighted negative factor or two or more other negative factors; medium risk $=$ at least one heavily weighted positive factor and at least one negative factor, or only one "other" negative factor; low risk $=$ no negative factors

income, $58.8 \%$ are low skilled, and $75.7 \%$ have low English proficiency. At the other end of the risk scale, the low-risk group is defined as having no negative factors at all. This group is also the most likely to have positive factors $(68.1 \%$ high household income, $27.7 \%$ working with high earnings, and $72.9 \%$ have private health insurance). Finally, the medium risk group includes everyone else not already classified in the high- and low-risk groups. It includes those who have a combination of negative and positive factors, or who have only one non-heavily-weighted negative factor but no positive factors. This medium-risk group is less likely to have heavily weighted negative factors and other negative factors than the high-risk group, and often has a positive factor $(52.3 \%$ have high household income and $53.7 \%$ have private health insurance). This three-tiered risk scale has broad categories that are simply intended to differentiate low, medium, and high risk. Due to the ambiguity of the Rules, it is difficult to predict the share of individuals within each risk category that would be deemed inadmissible.

\section{Regions of Birth}

We distinguish among the following regions of birth based on the respondent's place of birth: Mexicans and Central Americans, those from the Caribbean, South 
Americans, those from Middle East and Central Asia, ${ }^{6}$ sub-Saharan Africans, South/ East Asians, and we group those from Europe with countries that were colonized by Europeans (Canada and Oceania-i.e., mostly Australia and New Zealand) and where the majority of the population speaks English. For brevity, we refer to all groups other than the European/Canadian/Oceania/etc. as "non-European" and abbreviate the birth region of European/Canadian/Oceania/etc. as "ECO".

\section{Results}

\section{Disparate Impacts of the Public Charge Rules}

We first focus on which regional groups are at greatest risk of being deemed inadmissible by the new Public Charge rules. We examine the share with negative and positive factors by region in Table 3 , and the share at high, medium, and low risk according to our inadmissibility risk scale in Fig. 1.

With regard to the heavily weighted negative factors (Table 3), very few potential applicants have health conditions, and all non-European groups are either less likely than European, Canadian, and Oceanic (ECO) applicants to be economically inactive or are no different from them. However, all non-European groups are significantly more likely than ECO applicants to have other negative factors: low income, low skills, low English proficiency (except for sub-Saharan Africans), and residence in a large household. Applicants from Mexico/Central America stand out as particularly low income (37.8\%), low-skilled (53.1\%), low English proficient (64.2\%), and likely to live in large households $(23.0 \%)$. For ECO applicants, these figures are much lower: $13.9 \%, 8.8 \%, 16.5 \%$, and $6.5 \%$, respectively.

Non-European groups are also significantly less likely than potential applicants from ECO countries to have heavily weighted positive factors. Applicants from Mexico/Central America and the Caribbean stand out as among the least likely to have a household income greater than $250 \%$ of FPG, to work with earnings above $250 \%$ FPG, and to have private health insurance. South/East Asians are the most advantaged among the non-European groups, but they are still significantly less likely to have high household income, earnings, and private health insurance than ECO applicants.

On balance, most non-European groups, and especially Mexicans and Central Americans, are at much greater risk of receiving a public charge inadmissibility determination than ECO applicants. This is illustrated by the three-tiered risk scale shown in Fig. 1. All of the non-European groups are significantly more likely to be at the high-risk category of being deemed inadmissible, and significantly less likely to be in the low-risk category, compared with ECO applicants. Mexicans/Central

\footnotetext{
${ }^{6}$ Afghanistan, Armenia, Azerbaijan, Bahrain, Cyprus, Iran, Iraq, Israel, Jordan, Kuwait, Lebanon, Oman, Palestine, Gaza Strip, West Bank, Qatar, Saudi Arabia, Syria, Turkey, United Arab Emirates, Yemen, Pakistan, Republic of Georgia, Kazakhstan, Kirghizia, Tadzhik, Turkmenistan, Uzbekistan, Algeria, Egypt, Libya, Morocco, Sudan, Tunisia, and Western Sahara.
} 


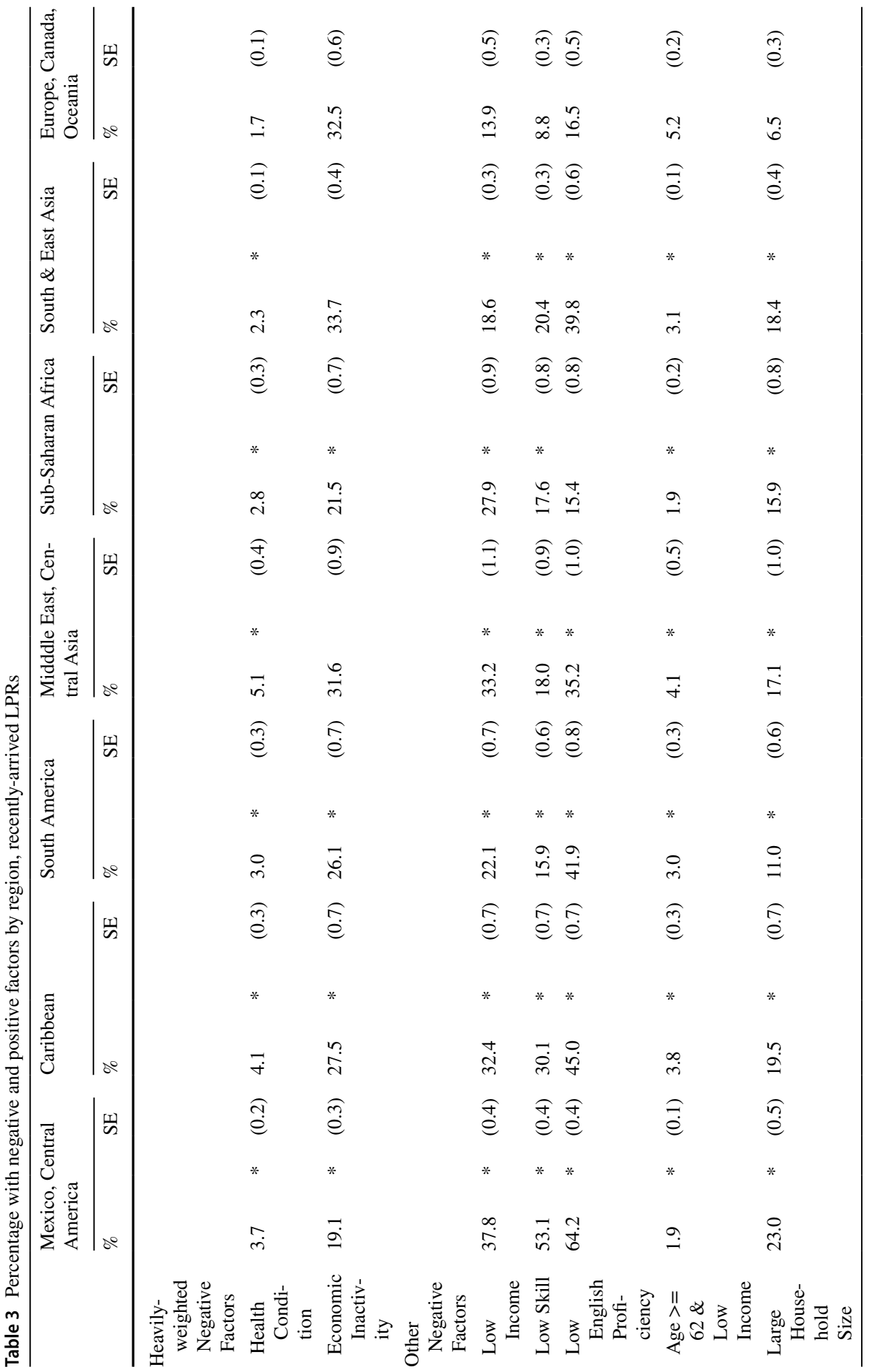




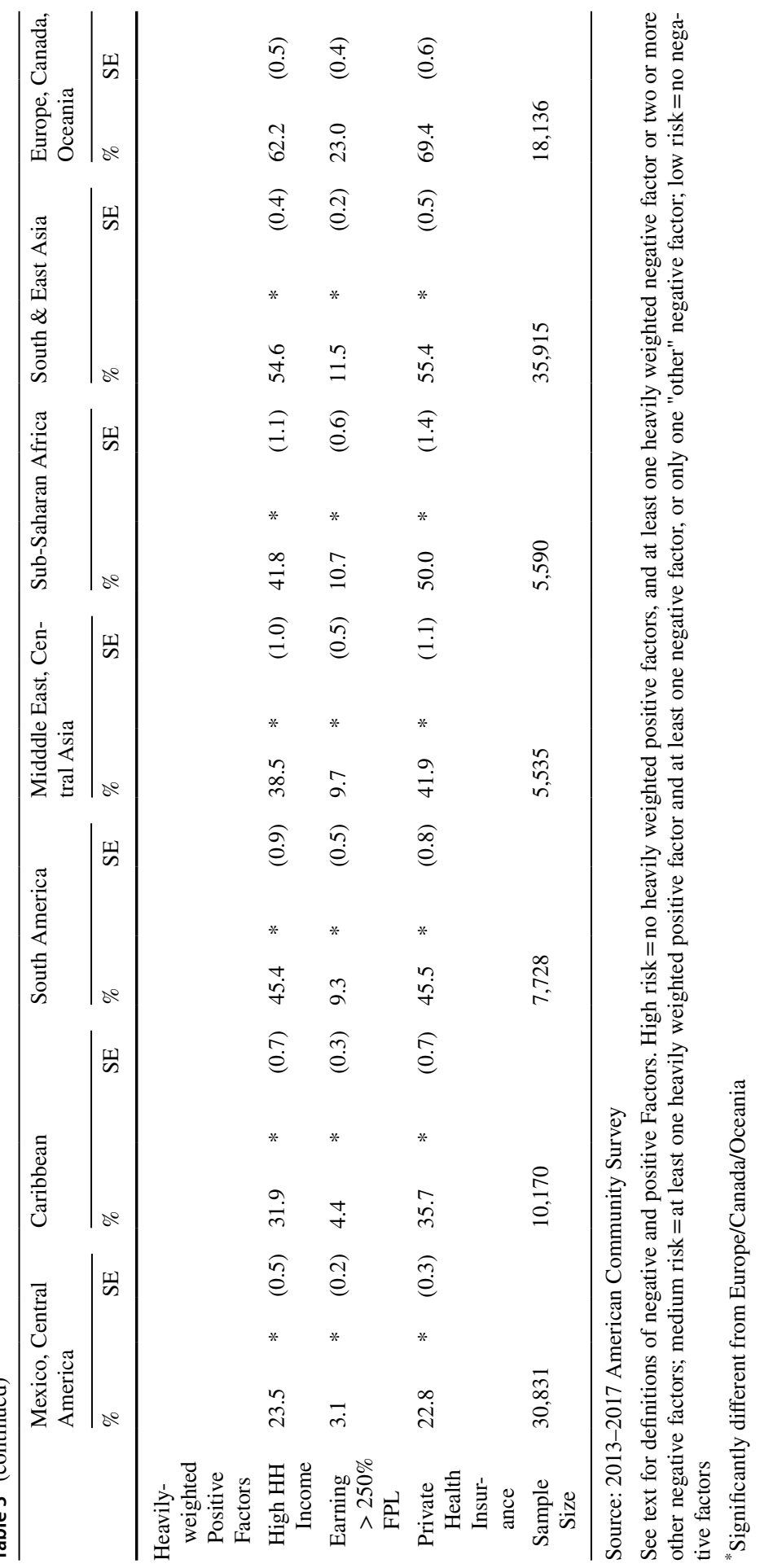




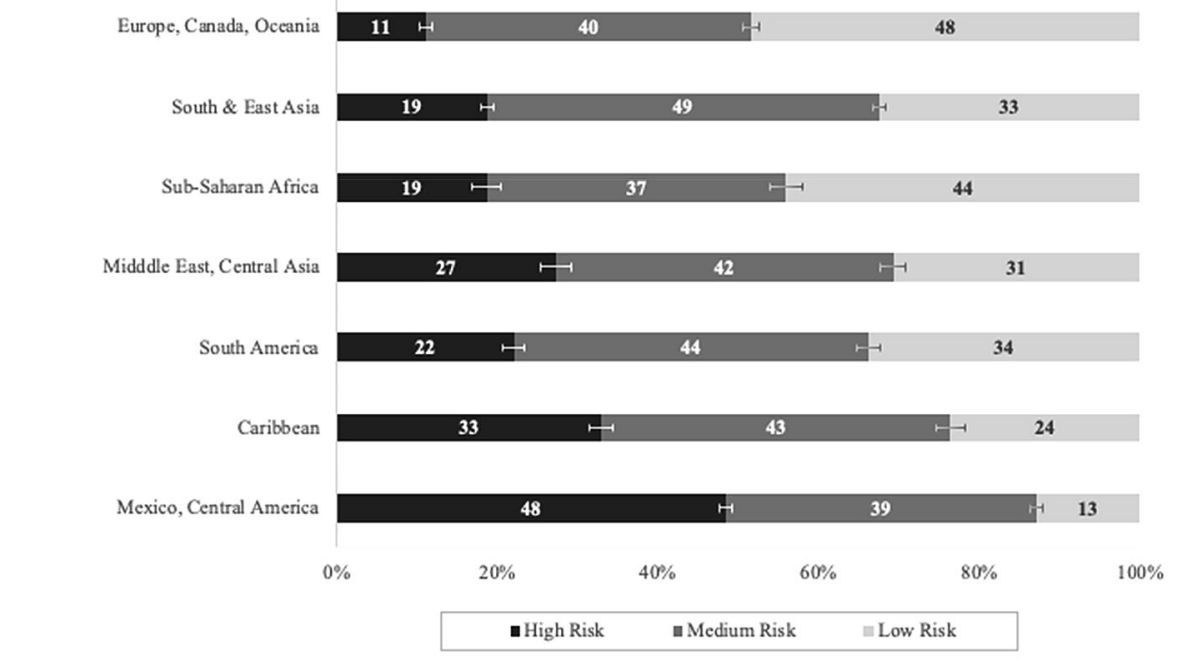

-High risk = No beavily weighted positive factorx, and at least 1 beavily weighted negative factor or 2 or more other negative faxtoos, Medium risk - At leass 1 beavily weightod pasitive factor and at least 1 negative factor, or only cees "ocher" negative factor; Low risk - no negative factors. Public program use is not included as a negative factor in this analysis, so these are conservative estimates $95 \%$ coefidence intervals are shown with the error bars.

Fig. 1 Estimated risk of being deemed inadmissible by the public charge rule* by region

Americans would face the highest risks under the Rule (an average of $48 \%$ are in the high-risk category and only $13 \%$ are in the low-risk category). Caribbean applicants also experience high risk of being deemed inadmissible; $33 \%$ are in the high-risk category, and only $24 \%$ are in the low-risk category. Applicants from South/East Asia experience lower risk (19\% are high-risk and 33\% are low-risk). Finally, ECO applicants face the lowest risk (11\% are high-risk and $48 \%$ are low-risk).

\section{The Risk of Inadmissibility and Welfare Use}

To address our second research question concerning whether inadmissibility risk is predictive of public benefits use, we examine the difference in the percentage using public benefits between the high- and low-risk group, referred to here as the "gradient," and we examine the positive predictive value of those who use public benefits. The positive predictive value indicates the percentage of those who are predicted to have some attribute through some type of screening process (i.e., to be at high risk of becoming a public charge) who actually have that attribute. We do not run regression models given that we aim to evaluate the Rules as they would be implemented by immigration officials who would use the positive and negative factors in a simpler analysis. Even with the limitations of the ACS measures, we expect to see a strong gradient and high positive predictive values if the negative and positive factors are valid predictors of the likelihood that an LPR applicant is more likely than not to be or become a public charge. Further, if there are disparities in the gradient or the positive predictive value, this would 
Table 4 Percentage Using Public Benefits by Three-Tiered Risk of Inadmissibility Scale by Region

\begin{tabular}{|c|c|c|c|c|c|c|c|c|}
\hline & \multicolumn{6}{|c|}{$\%$ Public benefit use } & \multirow[b]{2}{*}{ Gradient } & \multirow{2}{*}{$\begin{array}{l}\text { Positive } \\
\text { predictive } \\
\text { value }\end{array}$} \\
\hline & Low risk & SE & Med risk & $\mathrm{SE}$ & High risk & SE & & \\
\hline Mexico/Central America & 6.9 & $(0.4)$ & 9.9 & $(0.5)$ & 17.3 & $(0.9)$ & 10.4 & 17.3 \\
\hline Caribbean & 8.6 & $(0.7)$ & 20.4 & $(0.8)$ & 46.7 & $(1.5)$ & 38.0 & 46.7 \\
\hline South America & 7.9 & $(0.5)$ & 15.6 & $(1.0)$ & 34.6 & $(2.1)$ & 26.8 & 34.6 \\
\hline Middle East/Central Asia & 12.2 & $(0.6)$ & 33.1 & $(1.5)$ & 67.9 & $(1.9)$ & 55.6 & 67.9 \\
\hline Sub-Saharan Africa & 9.5 & $(0.7)$ & 20.6 & $(1.2)$ & 35.0 & $(2.6)$ & 25.6 & 35.0 \\
\hline South and East Asia & 6.9 & $(0.3)$ & 20.6 & $(0.5)$ & 60.2 & $(1.1)$ & 53.2 & 60.2 \\
\hline Europe/Canada/Oceania & 5.4 & $(0.3)$ & 15.8 & $(0.8)$ & 49.9 & $(2.5)$ & 44.5 & 49.9 \\
\hline
\end{tabular}

Source: 2013-2017 American Community Survey. LPRs and naturalized citizens who arrived or adjusted within last 5-10 years

See text for definitions of negative and positive Factors. High risk = no heavily weighted positive factors, and at least one heavily weighted negative factor or two or more other negative factors; medium risk $=$ at least one heavily weighted positive factor and at least one negative factor, or only one "other" negative factor; low risk = no negative factors

*Significantly different from Mexicans/Central Americans

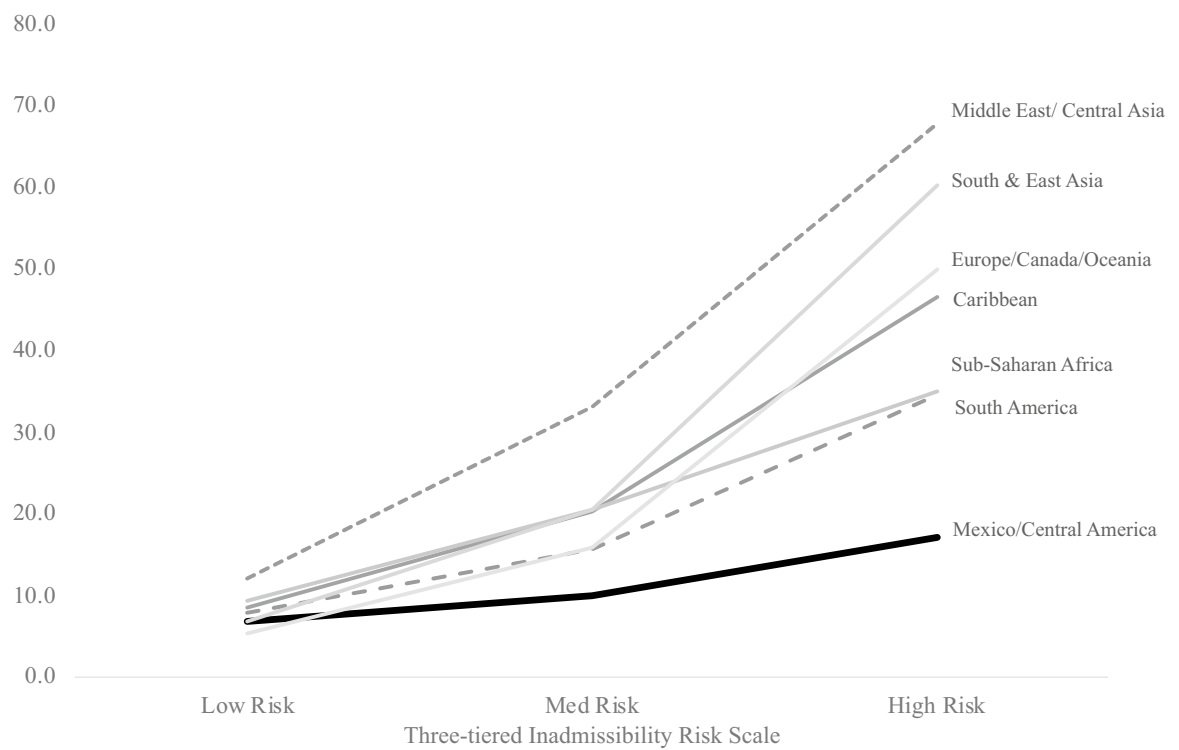

Fig. 2 percentage using public benefits, by risk of inadmissibility (three-tiered scale) and national origin

suggest that the positive and negative factors are more predictive for some groups than others.

For this analysis, we focused only on LPRs and naturalized citizens who arrived or adjusted status in the previous five to nine years. Those who were admitted in the last five years were excluded because they are ineligible for most federal public 
60

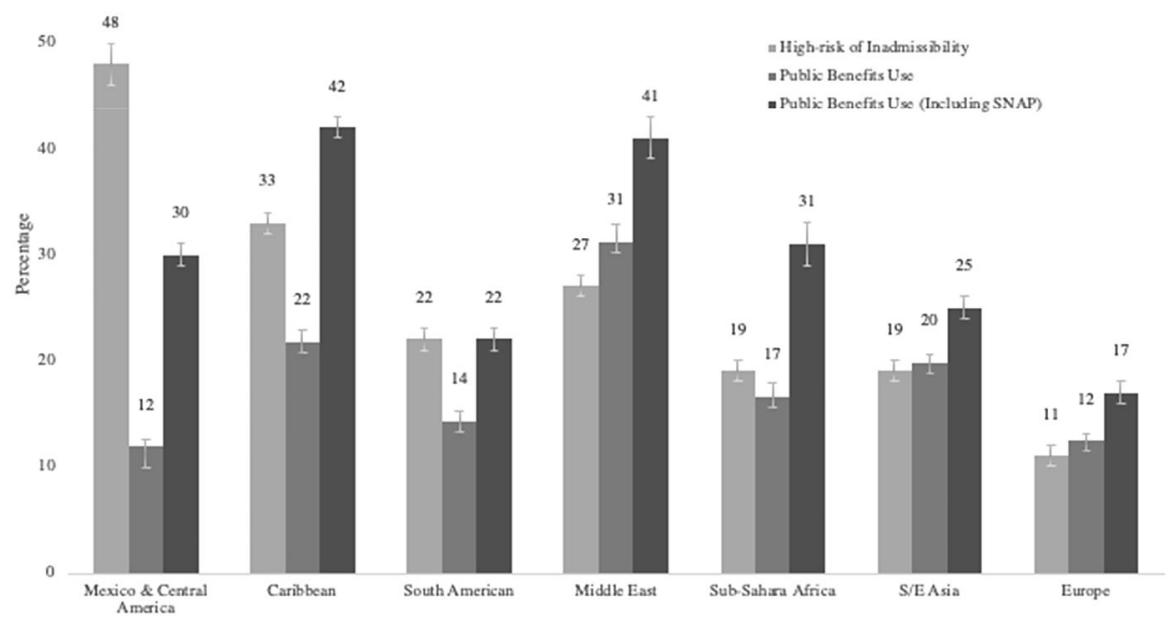

Fig. 3 Comparison of public benefits use and share in high-risk category, by region

benefits at the time of the ACS interview (although they are eligible for some statefunded programs). If we had included groups with limited eligibility for public benefits, this would have artificially depressed our estimates of the predictive validity. Public benefits use for these groups would be low regardless of their level of risk because they are ineligible for many public programs. Results are shown in Table 4 and Fig. 2.

Public benefit use increases across the risk categories (Table 4). In other words, the three-tiered inadmissibility risk scale is correlated with public benefits use. Nevertheless, for all regional groups except those from the Middle East and South/East Asia, fewer than half of those in the high-risk category received public benefits. Moreover, there is wide regional variation in the strength of the relationship. Mexicans/Central Americans have significantly weaker gradients (confirmed by $t$-tests in Table 4) than all other groups. Additionally, Mexicans/Central Americans have lower positive predictive values and are the least likely to use public benefits among those in the high-risk category (also see Fig. 2). T-tests show that the differences in positive predictive values between Mexicans/Central Americans and the other regional groups are all statistically significant.

An important implication of these results is that Mexicans and Central Americans could be at high risk of receiving a public charge determination even if their rate of public benefits use is not particularly high. To illustrate this point, we compared the share of each group of recently admitted LPRs in the high-risk category on the three-tiered inadmissibility risk scale with the share that use public benefits after living in the country 5-9 years.

We first consider receipt of programs that the ACS measures at the individual level: cash assistance programs (SSI and TANF) and Medicaid. Results are shown in Fig. 3. For most regional groups, the overall share that uses public benefits is within 


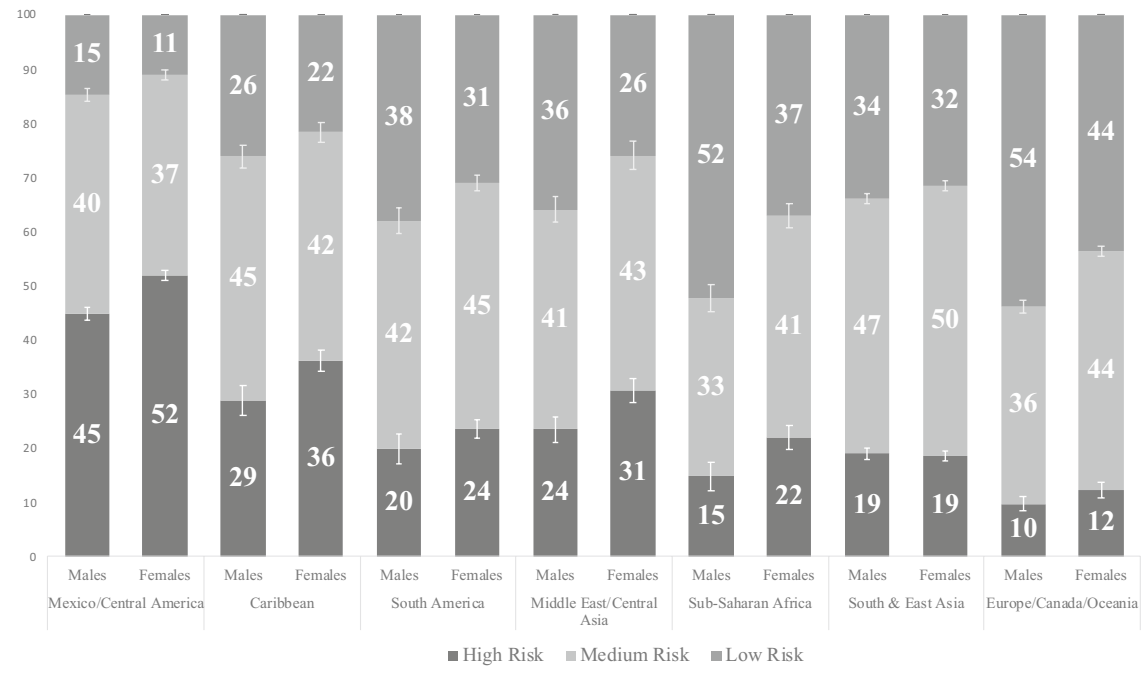

*High risk $=$ No heavily weighted positive factors, and at least 1 heavily weighted negative factor or 2 or more other negative factors; Medium risk = At least 1 heavily weighted positive factor and at least 1 negative factor, or only one "other" nega tive factor; Low risk = no negative factors. Public program use is not included as a negative factor in this analysis, so these are
conservative estimates. $95 \%$ confidence intervals are shown with the error bars.

Fig. 4 Estimated risk of being deemed inadmissible by the public charge rule* by gender and national origin

a few percentage points of the share in the high-risk group. However, nearly half of Mexicans/Central Americans are in the high-risk group even though only $12 \%$ use public benefits. When we add food assistance (SNAP), a program that the ACS measures at the household level, we obtain similar results. Although the share using public benefits increases for all groups when we add food assistance, Mexicans/Central Americans remain the only group for whom the share in the high-risk group (48\%) exceeds the share using public benefits (30\%). In additional analyses, we further considered family-level use of any public program (Medicaid, SNAP, or cash assistance), and obtained similar results. Overall, the findings suggest that the risk of being deemed inadmissible by the positive and negative factors is disproportionately high for Mexicans and Central Americans given their current levels of public benefit use.

\section{Supplemental Analyses}

We conducted several additional analyses to assess the robustness of the findings. First, we considered several alternative risk scales, including a simple sum of negative factors (similar to Capps, Greenberg, et al., 2018), the sum of negative factors for those who have no positive factors, and having no positive factor and at least one heavily weighted negative factor. Results, available upon request, were consistent regardless of the particular scale used.

Second, we benchmarked the ACS results to the SIPP to assess whether program usage differentials by race/ethnicity were consistent across data sources (SIPP has a 

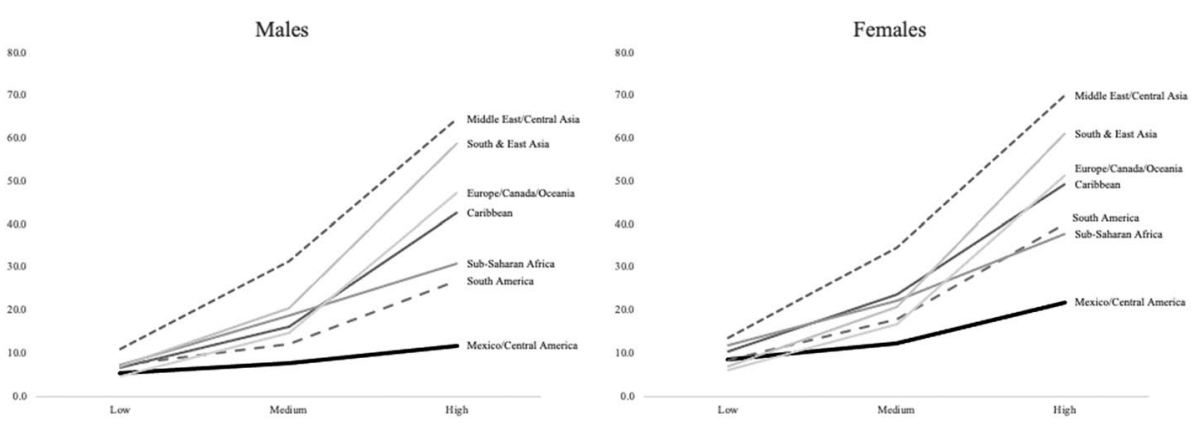

Fig. 5 Percentage using public benefits by gender, risk of inadmissibility (three-tiered scale), and region

smaller sample size so we did not rely on it for our main analyses). Because the SIPP does not include detailed region of birth information, we instead compared patterns by race/ethnicity. Rates of public benefits use tended to be higher in the SIPP than the ACS for all groups, and Hispanics were more likely to report Medicaid usage in the SIPP than the ACS. Therefore, the ACS-based results related to public benefits use are likely conservative. Regardless of data source, however, the patterns by race/ ethnicity were similar. While the share at high risk of inadmissibility was roughly equal to the share using public assistance among Asian, non-Hispanic white, and "other" non-Hispanic immigrants, the risk of inadmissibility was about 70 percent higher than the share using public assistance among Hispanics.

Third, we assessed whether the findings were consistent for men versus women. As shown in Fig. 1a, women were more likely to be deemed inadmissible than men for all regional groups other than South and East Asia. Women are also more likely to use public benefits (Fig. 2a). The gradient for public assistance use is steeper among females than men, as a larger share appears to use public programs, especially among the those in the highest risk category. Regardless of gender, however, the patterns by region of birth were the same. Both male and female immigrants from Mexico and Central America had the greatest risk of inadmissibility yet were the least likely to use public benefit programs among those at medium and high risk of inadmissibility (Figs. 4 and 5).

\section{Conclusion}

In efforts to analyze the impacts of the Public Charge rule, we utilize the American Community Survey (ACS) to determine whether the Rule would have disparate impacts on the risk of inadmissibility across region-of-birth groups, potentially altering the national composition of newly admitted legal immigrants. We also examine how effective the Rule is at identifying those who are most likely to use public assistance in the future. Consistent with prior research on leaked early versions of the Rule (Capps, Greenberg, et al., 2018), our analysis of the likely impacts of the policy suggests that the new Public Charge rules could dramatically reduce the number of LPRs from Mexico and Central America who are granted admission as legal 
permanent residents. We find that Mexican/Central Americans are at substantially higher risk for being deemed inadmissible under the Rule compared with European/ Canadian/Oceanic applicants. They are at higher risk because they are more likely to have multiple "other" negative factors (i.e., they are poorer, less well educated, and have lower English proficiency) and have few heavily weighted positive factors to offset these negative factors, and not because they are more likely to have heavilyweighted negative factors. Indeed, they are the least likely to be economically inactive among the seven regional groups we examined. Aside from Mexicans/Central Americans, other groups would also be impacted by the Rule, but to a lesser degree, including those from the Caribbean, South Americans, sub-Saharan Africans, and Middle Easterners and Central Asians. The risks faced by the non-European regions may be even higher than depicted here because the ACS does not permit us to measure all of the positive and negative factors, nor is it clear how many negative factors would be required for an applicant to be deemed inadmissible.

One of the consequences of these disparate impacts is that the number and national origin composition of newly admitted LPRs would likely change if the Rules were fully implemented. We anticipate that the Rules will reduce the number of poor and low-skilled LPRs admitted each year (many of whom originate from Mexico and Latin America), in part because of the additional processing time it will take immigration officials to implement the Rules, but also because fewer applicants will qualify. A back-of-the-envelope calculation suggests that if none of those in the "high-risk" category had been admitted over the last five years, the share of LPRs admitted from Mexico or Central America would have declined from 22 to $15 \%$. Nevertheless, we caution that the precise impact of the Rules is unclear. Some people may choose not to apply if they anticipate they will not qualify while the reverse may occur for other people. It is also possible that the Rules could reduce the waiting time for more socioeconomically advantaged applicants who are in line for employment or family-based visas while having little impact on the total numbers of admissions from a given world region.

We also find evidence that calls into question the rationale of the Rules as a tool for reducing immigrant welfare receipt. First, the Rules are inaccurate screening tools. In our simple descriptive analysis, we find that more than half of those who are at high risk of being deemed inadmissible do not use cash public assistance or Medicaid after living in the U.S. 5-10 years. Unfortunately, we were unable to follow LPRs longitudinally to assess whether they use public benefits later in life. Nevertheless, we speculate that if we were able to conduct such an analysis, the association between the positive and negative factors-which are measured at the time of admission - and public assistance use would weaken over time as immigrants adapt to the United States, learn English, and gain a foothold in the labor force.

Second, even though the Rules make no explicit reference to race, ethnicity, or national origin, the Rules are racially biased in their impacts. Mexicans/Central Americans have a much greater risk of being deemed inadmissible than any other regional group, yet their level of public assistance use is moderate compared with other, less disadvantaged, groups. Indeed, they were the only regional group for whom the share using public benefits was lower than the share in the high-risk group. This is true for both men and women, and whether we consider just the 
public programs we can attribute to individuals in the ACS (cash assistance and public health insurance), or whether we also add in the program that Mexicans and Central Americans' are the most likely to use to help feed their US-born children: food assistance. We also saw evidence of this pattern among Hispanics in both the ACS and SIPP.

Finally, regardless of region of birth, we found that women may be more impacted than men by the new Public Charge Rules. Female LPRs are more likely to be deemed inadmissible compared to their male counterparts, and they are more likely to use public assistance, meaning that they may be particularly vulnerable to pressure to avoid public programs on which they and their children depend. Women's greater usage of public programs could be related to use of food assistance as described above, or for prenatal care and childbearing in the case of Medicaid. Future research is needed to determine the cause of gender differences in program use and likelihood of inadmissibility.

These findings should be unsurprising because they are consistent with decades of past research showing relatively low rates of immigrant public assistance use, particularly among labor migrants from Mexico and Central America (e.g., Van Hook \& Bean, 2009b; Capps et al., 2009). Yet it is important to note that very little of the relevant research on immigrant public assistance use was discussed or cited in any of the official justification provided for the Public Charge rules (Inadmissibility on Public Charge Grounds, 2019). Although the purpose of the public charge rule is to restrict immigrant use of public assistance and further exclude immigrants who will use welfare, the Rule may lead to the exclusion of certain groups for reasons that are unrelated to their actual public benefits usage. Future policy should invest in appropriately measuring immigrant criteria for accurate and fair representation of risk. This is especially important because, as feared, participation in federal benefit programs has declined twice as fast among noncitizens relative to citizens between years 2016 and 2019, which is likely attributable to chilling effects resulting from fear surrounding the public charge rules and other restrictive policies (Capps, Gelatt, et al., 2020). This is especially problematic in light of the Covid-19 pandemic. Many immigrants are essential workers in jobs that do not offer health insurance. If they were to forgo public health care or other public benefits out of fear of the being deemed a public charge, this could contribute to noncompliance with COVID-19 mitigation guidelines (such as continuing to work even while sick), and an increased reluctance to getting tested, seeking medical care, or getting vaccinated, all of which could endanger public health.

Our study is not without limitations. As mentioned, the ACS is limited in that it does not allow us to focus directly on applicants at the point in time they would be evaluated by the Rules, namely when they are applying for adjustment to LPR status. In addition, we were unable to examine all measures of public benefit use that are consistent with those used by the Rule for making a public charge determination, namely individual participation in SNAP, public housing, or federal rental assistance programs. Notably important, the ACS public assistance/benefits measures do not capture participation in these programs during the 36-month period prior to when noncitizens applied for LPR status. Instead, the TANF and SSI measures pertain to the 12 months prior to the interview, and the Medicaid measure reflects current 
health insurance coverage (and is further limited by its grouping with other public health insurance benefits not at issue in the Rule).

However, we also conducted several sensitivity analyses and found that our findings were robust to alternative measures and specifications. First, it is unlikely that our results would change if we had been able to account for public program use in our measure of the risk of being deemed inadmissible, given that LPR applicants are very unlikely to have been eligible for federal public programs in the relevant time periods, an assumption we were able to confirm in our analysis of the 2008 SIPP. Second, we found that our conclusions are consistent regardless of how we summarized the negative and positive factors to measure the risk of inadmissibility (results also available upon request) or which data sources are used (SIPP or ACS) although Medicaid use among Hispanic groups was attenuated more than other groups. The Public Charge Rules are likely to raise the risk of inadmissibility among Mexicans and Central Americans more than other groups and despite their comparatively moderate levels of public assistance usage.

In closing, on February 2, 2021, the newly elected Biden Administration signed an Executive Order that requires a review of the implementation of the public charge ground of inadmissibility in Section 212(a)(4) of the Immigration and Nationality Act (INA), 8 U.S.C. 1182(a)(4), and the grounds of deportability [Section 237(a) (5) of the INA, 8 U.S.C. 1227(a)(5)]. This mandate will evaluate the current effects of the rules, identify appropriate agency actions to address immigration and public health concerns that stem from the rules, and recommend steps to reduce fear and confusion among immigrants in accessing government services (Biden, 2021). In the long run, President Biden could rescind the rules, however, this could take time. In the interim, the rules remain a debate of the courts, which have the potential to lead to a stay of, or a nationwide injunction. While we wait for action, applicants everywhere continue to comply with the Public Charge Rule. Our analysis should serve as a cautionary tale that the new public charge rules are blunt tools for use in immigration policy. As we have shown, they have the potential to impact Hispanic and female immigrant applicants at levels that are disproportionate to their actual costs to the United States.

Acknowledgement We acknowledge assistance provided by the Population Research Institute at Penn State University, which is supported by an infrastructure grant by the Eunice Kennedy Shriver National Institute of Child Health and Human Development (P2CHD041025) as well as the National Institutes of Health under the Social Environments and Population Health Training Program (T-32HD007514).

\section{References}

Alba, R. D., \& Nee, V. (2003). Remaking the American mainstream: Assimilation and the new immigration. . Cambridge, MA: Harvard University Press.

Arthur, A. R. (2018). Two reasons to amend the public charge ground of inadmissiblity: Protecting the taxpayers and the disadvantaged. . Washington, DC: Center for Immigration Studies.

Bachmeier, J. D., Van Hook, J., \& Bean, F. D. (2014). Can we measure immigrants'? Legal status lessons from two U.S. surveys. International Migration Review, 48(2), 538-566. https://doi.org/10.1111/ imre.12059. 
Batalova, J., Fix, M., \& Greenberg, M. (2018). Chilling effects: The expected public charge rule and its impact on legal immigrant families' public benefits use. Washington, DC: Migration Policy Institute. Available online at https://www.migrationpolicy.org/research/chilling-effects-expected-publiccharge-rule-impact-legal-immigrant-families

Batalova, J., Hooker, S., \& Capps, R. (2014). DACA at the two-year mark: A national and state profile of youth eligible and applying for deferred action. Washington, DC: Migration Policy Institute. Available online at https://www.migrationpolicy.org/research/daca-two-year-mark-national-and-state-profi le-youth-eligible-and-applying-deferred-action

Bean, F. D., Van Hook, J. V. W., \& Glick, J. E. (1997). Country of origin, type of public assistance and patterns of welfare recipiency among U.S. immigrants and natives. Social Science Quarterly, 78(2), $432-451$.

Bell, S. H. (2001). Why are welfare caseloads falling. . Washington, DC: The Urban Institute.

Biden, J. R. (2021). Executive order on restoring faith in our legal immigration systems and strengthening integration and inclusion efforts for new Americans. The White House. Retrieved March 4, 2021, from https://www.whitehouse.gov/briefing-room/presidential-actions/2021/02/02/executiveorder-restoring-faith-in-our-legal-immigration-systems-and-strengthening-integration-and-inclu sion-efforts-for-new-americans/.

Borjas, G. J. (1990). Friends or strangers: The impact of immigrants on the US economy. . New York: Basic Books.

Borjas, G. J., \& Hilton, L. (1996). Immigration and the welfare state: Immigrant participation in meanstested entitlement programs. The Quarterly Journal of Economics, 111(2), 575-604.

Capps, R. (2001). Hardships among children of immigrants: Findings from the 1999 National Survey of America's Families. Assessing the New Federalism Policy Brief Series B, No. B-29, The Urban Institute, Washington, DC.

Capps, R., Bachmeier, J. D., \& Van Hook, J. (2018b). Estimating the characteristics of unauthorized immigrants using U.S. census data: Combined sample multiple imputation. The Annals of the American Academy of Political and Social Science, 677(1), 165-179. https://doi.org/10.1177/00027 16218767383.

Capps, R., Fix, M., \& Batalova, J. (2020). Anticipated "Chilling Effects" of the public-charge rule are real: Census data reflect steep decline in benefits use by immigrant families. Migrationpolicy.Org. https://www.migrationpolicy.org/news/anticipated-chilling-effects-public-charge-rule-are-real

Capps, R., Fix, M., \& Henderson, E. (2009). Trends in Immigrants' use of public assistance after welfare reform. In M. Fix (Ed.), Immigrants and welfare: the impact of welfare reform on America's newcomers. (pp. 123-152). New York: Russell Sage Foundation.

Capps, R., Gelatt, J., Soto, A. G. R., \& Van Hook, J. (2020). Unauthorized immigrants in the United States: Stable numbers, changing origins. Washington, DC: Migration Policy Institute. https://www. migrationpolicy.org/sites/default/files/publications/mpi-unauthorized-immigrants-stablenumberschangingorigins_final.pdf.

Capps, R., Greenberg, M., Fix, M., \& Zong, J. (2018). Gauging the impact of DHS' proposed publiccharge rule on U.S. immigration. Washington, DC: Migration Policy Institute. https://www.migra tionpolicy.org/research/impact-dhs-public-charge-rule-immigration.

Chiswick, B. R., \& Miller, P. W. (2002). Immigrant earnings: Language skills, linguistic concentrations and the business cycle. Population Economics, 15, 31-57.

Faber, A. S. (2018). A vessel for discrimination: The public charge standard of inadmissibility and deportation. The Georgetown Law Journal, 108, 34.

Fix, M. E., \& Passel, J. S. (1999). Trends in noncitizens' and citizens' use of public benefits following welfare reform. . Washington, DC: The Urban Institute.

Fomby, P., \& Cherlin, A. J. (2004). Public assistance use among U.S.-born children of immigrants. International Migration Review, 38(2), 584-610. https://doi.org/10.1111/j.1747-7379.2004.tb00210.x.

Fortuny, K., \& Chaudry, A. (2012). Overview of immigrants' eligibility For SNAP, TANF, Medicaid, and CHIP. . Washington, DC: US Department of Health and Human Services, Office of the Assistant Secretary for Planning and Evaluation, Office of Human Services Policy.

Inadmissibility on Public Charge Grounds, 84 Fed. Reg. 157 (August 14, 2019) (to be codified at 8 CFR Parts 103, 212, 213, 214, 245 and 248)

Kaushal, N. (2005). New immigrants' location choices: magnets without welfare. Journal of Labor Economics, 23(1), 59-80.

Lofstrom, M., \& Bean, F. D. (2002). Assessing immigrant policy options: Labor market conditions and postreform declines in immigrants' receipt of welfare. Demography, 39(4), 617-638. 
Meyer, B. D., Mok, W. K.C., \& Sullivan, J. X. (2009). The under-reporting of transfers in household surveys: Its nature and consequences. NBER Working Paper No. 15181, Cambridge, MA: National Bureau of Economic Research.

Myers, D., \& Pitkin, J. (2010). Assimilation today: New evidence shows the latest immigrants to America are following in our history's footsteps. Washington: Center for American Progress. Available online at https://cdn.americanprogress.org/wp-content/uploads/issues/2010/09/pdf/immigrant_ assimilation.pdf

Page, K. R., Venkataramani, M., Beyrer, C., \& Polk, S. (2020). Undocumented U.S. immigrants and Covid-19. New England Journal of Medicine. https://doi.org/10.1056/NEJMp2005953.

Perreira, K. M., Yoshikawa, H., \& Oberlander, J. (2018). A new threat to immigrants' health-The public-charge rule. New England Journal of Medicine, 379(10), 901-903. https://doi.org/10.1056/ NEJMp1808020.

Richwine, J. (2018). Legitimizing the public charge determination: Comment on 'inadmissibility on public charge grounds. DHS Docket No. USCIS-2010-0012. Center for Immigration Studies, Washington, DC.

Ruggles, S., Flood, S., Goeken, R., Grover, J., Meyer, E., Pacas, J., \& Sobek, M. (2020). IPUMS USA; Version 10.0 [dataset]. Minneapolis, MN: IPUMS. https://doi.org/https://doi.org/10.18128/D010. V10.0

Van Hook, J., Bachmeier, J. D., Coffman, D., \& OferHarel, O. (2015). Can we spin straw into gold? An evaluation of immigrant legal status imputation approaches. Demography, 52(1), 329-354. https:// doi.org/10.1007/s13524-014-0358-x.

Van Hook, J., \& Bean, F. D. (2009). Immigrant welfare receipt: Implications for immigrant settlement and integration. In M. Fix (Ed.), Immigrants and welfare: the impact of welfare reform on America's newcomers. (pp. 93-122). New York: Russell Sage Foundation.

Van Hook, J., \& Bean, F. D. (2009). Explaining Mexican-immigrant welfare behaviors: The importance of employment-related Cultural repertoires. American Sociological Review, 74(3), 423-444. https:// doi.org/10.1177/000312240907400305.

Warren, R. (2014). Democratizing data about unauthorized residents in the United States: Estimates and public-use data, 2010 to 2013. Journal on Migration and Human Security, 2(4), 305-328.

Publisher's Note Springer Nature remains neutral with regard to jurisdictional claims in published maps and institutional affiliations. 